\title{
Spectral-Based Analysis of Progressive Dynamical Changes in the Fetal Heart Rate Signal During Labor by Using Empirical Mode Decomposition
}

\author{
Patricio Fuentealba ${ }^{1,2}$, Alfredo Illanes ${ }^{1}$, Frank Ortmeier $^{1}$ \\ ${ }^{1}$ Otto-von-Guericke University, Magdeburg, Germany \\ ${ }^{2}$ Universidad Austral de Chile, Valdivia, Chile
}

\begin{abstract}
In this work, we propose to study the progressive fetal response along the fetal heart rate (FHR) signal by using empirical mode decomposition and time-varying spectralbased analysis. The main idea is to investigate if a particular FHR signal episode in the time-domain reflects $d y$ namical changes in the frequency-domain that can help to assess the fetal condition. Results show that the spectral components associated with the neural sympathetic fetal reactivity exhibit significant spectral energy differences between normal and acidotic fetuses.
\end{abstract}

\section{Introduction}

Timely identification of fetuses with the risk of asphyxia during labor enables clinicians to prevent potential adverse outcomes and without excessive intervention [1]. This procedure is usually based on the analysis of fetal heart rate (FHR) and uterine contraction (UC) signals obtained through the Cardiotocograph (CTG). However, the CTG analysis is difficult because it involves the interpretation of highly complex signals, whose methodology has shown to lack objectivity and poor reproducibility [1].

In order to improve the CTG interpretation, different medical guidelines [2] and computer-based support (CS) [3] have been proposed. However, concerning to those methods, guidelines lack consensus in several aspects and it has not been proven that CS improve the results so far.

On the other hand, recent literature indicates that each fetus has its own control and that its condition depends on how the fetus is compensating itself over time [4]. Likewise, experienced clinicians attempt to consider this evolution when interpreting CTGs by considering a specific FHR characteristic along a temporal window [2]. Under this concept, it seems that methods that do not consider these characteristics could not be appropriated for a correct analysis, since the interpretation is based just on a snapshot of the complete nonstationary input-output process [5].

In this context, several approaches based on time-varying signal processing techniques have been proposed [1] such as Short Time Fourier Transform, quadratic time-frequency distributions or time-varying autoregressive (AR) modeling. Likewise, Continuous and Discrete Wavelets Transform techniques have been proposed in order to analyze the transient nature of the UC excitation. However, most of them are mainly focused on fetal reactivity as a response to a $\mathrm{UC}$, without taking into account the progressive spectral variations from one event to another.

In this work, we propose to analyze such progressive characteristics along the FHR signal by using the complete ensemble empirical mode decomposition with adaptive noise (CEEMDAN) method [6] and parametric time-varying spectral-based analysis. The main idea is to study the spectral progressive FHR dynamics that can help to assess the fetal condition.

Results show that the CEEMDAN mode associated with the neural sympathetic fetal reactivity band (0.03$0.15 \mathrm{~Hz}$ ) exhibits significant spectral energy differences $(p$ value $<0.02$ ) between normal and acidotic cases.

\section{Methodology}

The main idea behind the proposed method is to study spectral progressive variations present in the FHR signal that can be related to the fetal condition. For this purpose, we propose a modal spectral-based analysis performed by using CEEMDAN [6] and time-varying AR modeling.

\subsection{FHR signal pre-processing}

The FHR and UC signals acquisition is commonly subjected to different types of artifacts such as loss of data and outliers. Hence, following [7], FHR signal values outside of range between $50 \mathrm{bpm}$ and $200 \mathrm{bpm}$ are removed, and then loss of data less than $75 \mathrm{~s}$ length are interpolated by using a Hermite spline method. Likewise, UC loss of data less than $25 \mathrm{~s}$ are interpolated and filtered by a moving average filter of $15 s$ windows length. This filtered UC signal is using for the decelerations identification. 
In the sequel, the preprocesed FHR and UC signals are denoted simply as the FHR and UC signal, respectively.

\subsection{Decelerations identification}

The decelerations identification is performed following [8]. In a first step, the evident segments are recognized by a floating-line and a progressive baseline, which are computed by a nonlinear median filter [9] over a sliding window of $10 \mathrm{~s}$ and $400 \mathrm{~s}$ length, respectively [8].

In a second step, the segments that were not recognized as evident decelerations, but they are certainly a response due to a UC, are identified. Those episodes (UC-seg) are identified according to the criteria defined in [10], where a UC-seg starts $7 s$ before the UC apex and ends $50 s$ after it.

\subsection{FHR signal decomposition}

This work aims to study the spectral characteristics associated with the neural sympathetic fetal reactivity modulated by the autonomic nervous system (ANS), which mainly lie in the frequency band between 0.03 and $0.15 \mathrm{~Hz}$ [1]. Hence, firstly it is necessary to attenuate the very low frequency $(0$ and $0.03 \mathrm{~Hz})$, associated with the morphological characteristics of FHR decelerations. Following [8], this filtered FHR signal is computed by the detrending between the FHR signal and the floating-line.

For the subsequent analysis, the filtered FHR signal is decomposed by using the CEEMDAN method [6]. It allows to decompose nonlinear and non-stationary signals into a finite number of components. Its main advantage is that it depends on local properties of the signal itself. In consequence, it does not require a priori information as other methods such as wavelet and Fourier transform. For more details of the CEEMDAN method, please refer to [6].

In the sequel, the CEEMDAN components extracted from the FHR signal are denoted as the FHR modes.

\subsection{Time-varying AR spectrum estimation}

For the spectral analysis of each FHR mode, the time-varying AR modeling is used, since this method offers certain advantages over other standard spectral-based methods [11]. It allows the extraction of quantitative spectral parameters versus time and requires only a fraction of the samples needed by standard techniques (e.g. Fast Fourier transform) to obtain the same resolution.

The AR time-varying spectrum can be described by:

$$
S_{A R}[f, n]=\frac{1}{\left|1+\sum_{k=1}^{p} a_{k}(n) e^{-j 2 \pi f k}\right|^{2}}
$$

where $p$ is the model order and $a_{k}$ are the AR parameters.

Following [10], the order $p$ was set to $6^{\text {th }}$ and the AR coefficients $a_{k}(n)$ were computed by using a recursive least squares algorithm with a forgetting factor set to 0.99 . For more information of AR modeling, please refer to [11].

\section{Results}

The analysis is performed using data extracted from the CTU-UHB Intrapartum Cardiotocography database [12]. It contains $552 \mathrm{CTG}$ recordings sampled at $4 \mathrm{~Hz}$. Codes have been implemented in Matlab ${ }^{\circledR}$ version 2015b.

For the evaluation, a dataset of CTG recordings was selected according to their outcome parameters of $\mathrm{pH}$ and BDecf values. Values of $\mathrm{pH}<7.05$ and BDecf $\geq 12$ commonly indicate a fetal acidosis, whereas $\mathrm{pH}$ values between 7.20 and 7.60 indicate a normal fetal condition [13]. Therefore, CTG recordings labeled by values of $\mathrm{pH}<7.05$ and BDecf $\geq 12$ were selected as examples of acidotic fetuses and recordings labeled by $\mathrm{pH}>7.35$ (arbitrarily chose from the normal range) and $\mathrm{BDecf}<12$ were selected as examples of normal fetuses. Under this criteria, 60 recording were selected for the analysis, 18 correspond to acidotic cases and 42 cases correspond to normal cases.

According to [1], the frequency band associated with neural sympathetic fetal reactivity lie in the range between 0.03 and $0.15 \mathrm{~Hz}$. Therefore, the FHR mode whose spectral dynamics are inside this frequency range was studied.

The Fig.1 exhibits two representative examples, one for each column. The first (left) corresponds to a normal case and the second (right) to an acidotic case, belonging to the recording $1189 \mathrm{~m}$ and $1104 \mathrm{~m}$, respectively. The first row shows the raw FHR signal. The second row depicts the FHR decelerations (black), floating-line (blue) and the progressive baseline (magenta). The third row plots the FHR mode of interest $\left(6^{t h}\right)$. The fourth row exhibits the AR spectrum computed from the FHR mode, whose values were normalized between 0 and 1 for each sample $n$.

In the fifth row the spectral energy $(E)$ is plotted (values were normalized between 0 and 100). It is calculated from the total frequency band $(0-2 \mathrm{~Hz})$ of the AR spectrum for each sample $n$ as described in eq. (2).

$$
E[n]=\sum_{f=0}^{2 H z}\left(S_{A R}[f, n]\right)
$$

Finally, the last row depicts the average of the $E$ during decelerations $(E D D)$ in red markers. In Fig.1(e,f,i-l) the deceleration segments are highlighted in gray.

Results show that the analyzed cases exhibit different spectral behavior. Both cases show important spectral dynamics over time, which differ between an acidotic and a normal condition. Particularly, in the first example presented in the Fig.1 (left), the AR spectrum exhibits different spectral dynamical changes, whose $E$ (Fig.1(i)) describes pronounced variations in amplitude. 

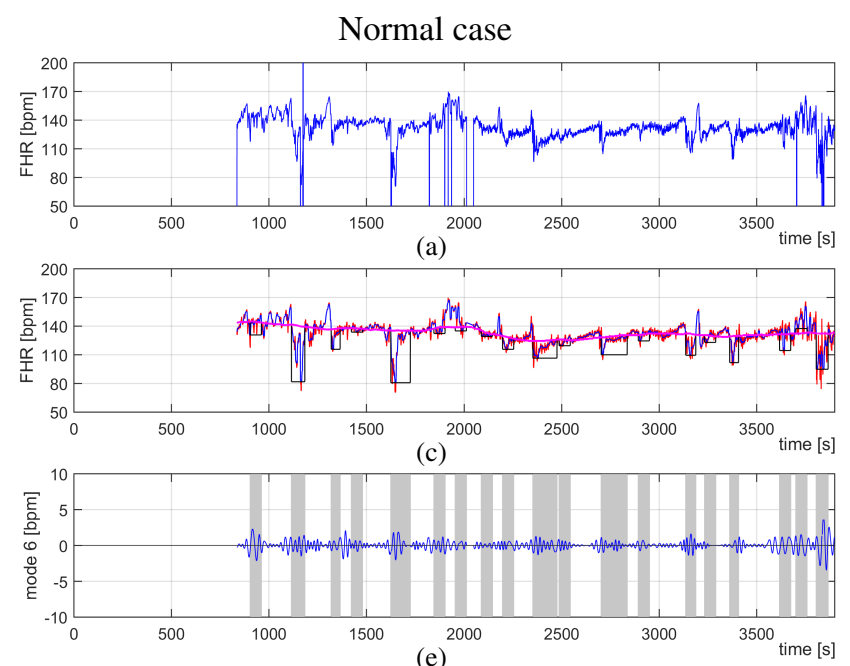

(e)
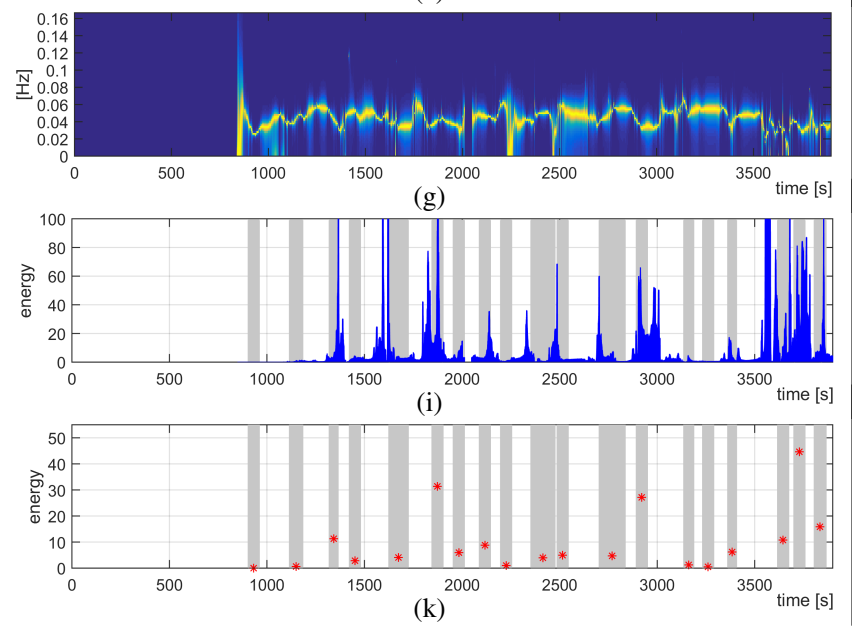
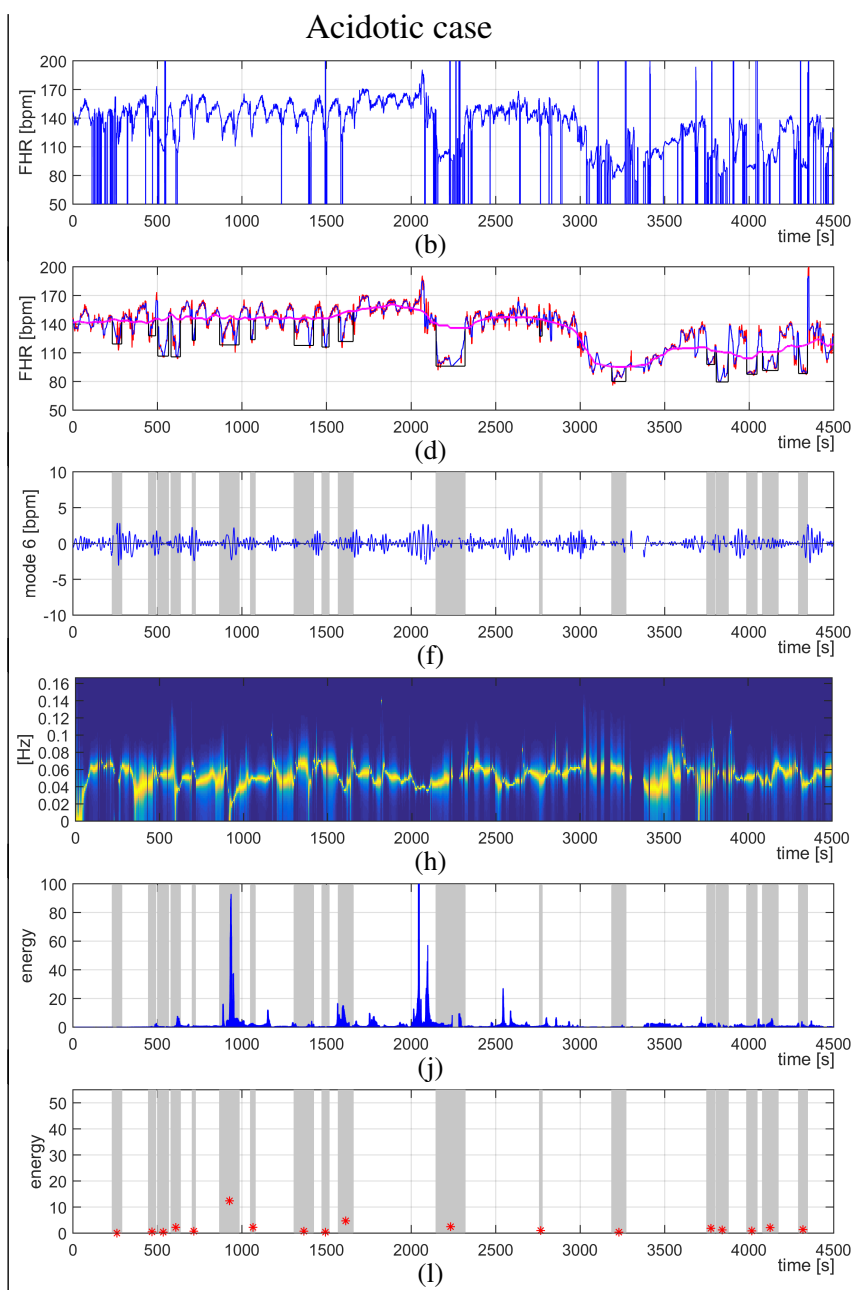

Figure 1: (a) Raw FHR signal nb. $1189 \mathrm{~m}, \mathrm{pH}=7.36$ and $\mathrm{BDecf}=0.43$; (b) Raw FHR signal nb. $1104 \mathrm{~m}, \mathrm{pH}=6.92$ and $\mathrm{BDecf}=23.75$; (c-d) FHR signal (red), floating-line (blue), progressive baseline (magenta) and decelerations (black); (e-f) FHR mode $6^{\text {th }}$; (g-h) time-varying AR spectrum; (i-j) spectral energy $E$; (k-1) EDD (red markers).

In contrast to the previous example, the acidotic case shows a completely different spectral behavior (see Fig.1(j)). In this case, the $E$ does not show prominent variations, i.e. the AR spectrum exhibits a less marked response in the $E$ compared to the first case. Likewise, its $E$ level is considerably lower compared to the first case.

This phenomenon can be also observed in the EDD, which is plotted in the last row of Fig.1. Here, we can clearly observe that the $E D D$ is in general higher for the normal than the acidotic case. Likewise, for the acidotic case the $E D D$ shows a more stable behavior from one deceleration to another compared to the normal case.

Likely, this phenomenon can be related to the fetal condition since the fetal reactivity, modulated by the sympathetic ANS, increases for a normal fetus compared to an acidotic fetus [10]. Therefore the neural sympathetic fetal reactivity of an acidotic fetus might not reflect high activity in the FHR signal compared with a normal fetus.

In order to prove if this phenomenon is reflected in the other signals of the dataset, we compute two features: the average of $E(\bar{E})$ and the average of $E D D(\overline{E D D})$. Then a Wilcoxon rank-sum test was employed to evaluate if these features show a statistically significant difference between the normal and acidotic cases, performed under the hypothesis that the median values of the features differ between normal and acidotic cases.

The obtained results are presented in Table 1, whose boxplots are exhibit in Fig.2. Here, we can observe that the median values of $\bar{E}$ are 3.45 and 2.24 and the median values of $\overline{E D D}$ are 3.62 and 2.50 for the normal and acidotic cases, respectively. From the statistical test, the values of both features were significantly higher $(p$-value $<0.02)$ for the group of normal cases compared to the acidotic cases. As a result, the proposed hypothesis has been proven. 
Table 1: Analysis of extracted spectral features

\begin{tabular}{cccc}
\hline \hline & $\begin{array}{c}\text { Normal } \\
\text { cases }\end{array}$ & $\begin{array}{c}\text { Acidotic } \\
\text { cases }\end{array}$ & $\begin{array}{c}\text { Significance } \\
(p \text {-value })\end{array}$ \\
\hline $\bar{E}$ & $3.45[2.56-6.61]$ & $2.24[2.07-3.24]$ & $<0.02$ \\
$\overline{E D D}$ & $3.62[2.54-6.56]$ & $2.50[1.80-3.37]$ & $<0.02$ \\
\hline \hline
\end{tabular}

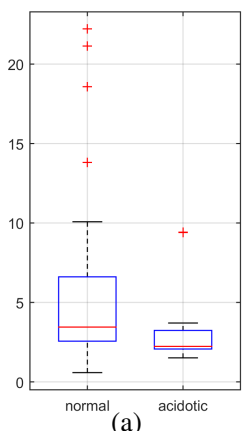

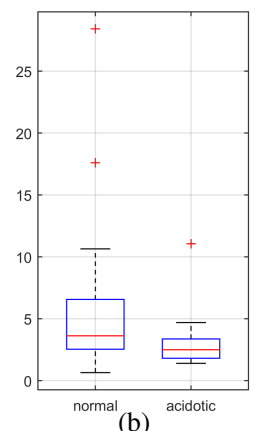

Figure 2: Boxplots for normal and acidotic cases of the selected dataset; (a) feature $\bar{E}$; (b) feature $\overline{E D D}$; median of the data (red). The borders of the box are the $25 \mathrm{th}$ and $75 t h$ percentiles of the data and red crosses are the outliers.

\section{Conclusion}

The obtained results showed that CEEMDAN method in combination with the time-varying AR modeling can be a powerful methodology for the CTG signals assessment. In fact, the analysis of the spectral components associated with the neural sympathetic fetal reactivity $(0.03-0.15 \mathrm{~Hz})$ allowed to recognize significant differences $(p$-value $<0.02)$ in the $E$ between normal and acidotic fetuses, represented by the features $\bar{E}$ and $\overline{E D D}$.

The $E D D$ from one FHR deceleration to another over time, strongly differ between a normal and an acidotic case, presenting higher variations for a normal case. Considering that FHR decelerations are one of the most complex patterns to assess, these results open perspectives for the characterization of them based on this methodology, in order to improve the interpretation and subsequent classification of non-reassuring CTG recordings.

As a future step, beside the proposed features, we propose to extract a greater number of them and evaluate their performance by an automatic CTG classification.

\section{Acknowledgements}

Research funded by the National Commission for Scientific and Technological Research CONICYT, Chile.

\section{References}

[1] Haritopoulos M, Illanes A, Nandi AK. Survey on cardiotocography feature extraction algorithms for foetal wel- fare assessment. In XIV Mediterranean Conference on Medical and Biological Engineering and Computing 2016. Springer, 2016; 1193-1198.

[2] Ayres-de Campos D, Spong CY, Chandraharan E, et al. Figo consensus guidelines on intrapartum fetal monitoring: Cardiotocography. International Journal of Gynecology Obstetrics 2015;131(1):13-24.

[3] Nunes I, Ayres-de Campos D. Computer analysis of foetal monitoring signals. Best Practice Research Clinical Obstetrics Gynaecology 2016;30:68-78.

[4] Ugwumadu A. Are we (mis) guided by current guidelines on intrapartum fetal heart rate monitoring? case for a more physiological approach to interpretation. BJOG An International Journal of Obstetrics Gynaecology 2014; 121(9):1063-1070.

[5] Warrick PA, Hamilton EF, Precup D, Kearney RE. Classification of normal and hypoxic fetuses from systems modeling of intrapartum cardiotocography. IEEE Transactions on Biomedical Engineering 2010;57(4):771-779.

[6] Colominas MA, Schlotthauer G, Torres ME. Improved complete ensemble emd: A suitable tool for biomedical signal processing. Biomedical Signal Processing and Control 2014;14:19-29.

[7] Spilka J, Georgoulas G, Karvelis P, Oikonomou VP, Chudáček V, Stylios C, Lhotská L, Janku P. Automatic evaluation of fhr recordings from ctu-uhb ctg database. In Information Technology in Bio-and Medical Informatics. Springer, 2013; 47-61.

[8] Fuentealba P, Illanes A, Ortmeier F. Progressive fetal distress estimation by characterization of fetal heart rate decelerations response based on signal variability in cardiotocographic recordings. Computing in Cardiology 2017;44:1.

[9] Sameni R, Shamsollahi M, Jutten C. Model-based bayesian filtering of cardiac contaminants from biomedical recordings. Physiological Measurement 2008;29(5):595.

[10] Romano M, Bifulco P, Cesarelli M, Sansone M, Bracale M. Foetal heart rate power spectrum response to uterine contraction. Medical and Biological Engineering and Computing 2006;44(3):188-201.

[11] Cazares S, Moulden M, Redman CW, Tarassenko L. Tracking poles with an autoregressive model: a confidence index for the analysis of the intrapartum cardiotocogram. Medical engineering physics 2001;23(9):603-614.

[12] Chudáček V, Spilka J, Burša M, Janku P, Hruban L, Huptych M, Lhotská L. Open access intrapartum CTG database. BMC pregnancy and childbirth 2014;14(1):16.

[13] Kumar N, Suman A, Sawant K. Relationship between immediate postpartum umbilical cord blood ph and fetal distress. International Journal of Contemporary Pediatrics 2016;3(1):113-119.

Address for correspondence:

Patricio Fuentealba

Faculty of Computer Science

Universitätsplatz 2, 39106, Magdeburg, Germany

patricio.fuentealba@ovgu.de 